- Focuses on a condition that is being encountered with increasing frequency.

- Highlights the importance of considering osteonecrosis as a differential diagnosis for orofacial pain.

- Suggests possible management of osteonecrosis.

\title{
Orofacial pain - a presenting symptom of bisphosphonate associated osteonecrosis of the jaws
}

\author{
D. Srinivasan, ${ }_{1}^{1}$ S. Shetty, ${ }_{1}^{2}$ D. Ashworth, ${ }^{3}$ N. Grew ${ }^{4}$ and B. Millar ${ }^{5}$
}

Osteonecrosis of the jaws (ONJ) associated with bisphosphonate therapy is being reported with increasing frequency all over the world. Pain may be the presenting symptom commonly associated with intra-oral bone exposure. The aetiology of this therapeutic complication is still not well understood. Three cases are presented and the management of the condition is discussed.

\section{INTRODUCTION}

Bisphosphonates are frequently used to stabilise skeletal metastatic deposits and increasingly for neo-adjuvant prophylaxis in younger breast cancer patients. They are also used in the management of hypercalcaemia secondary to malignancy. It is commonly administered intravenously for this purpose. The oral administered drugs can be used for the above but are more commonly prescribed for the prevention or treatment of osteoporosis in post menopausal women and corticosteroid induced osteoporosis.

One of the actions of bisphosphonates is to interrupt the mevalonate pathway, resulting in osteoclast apoptosis. This interferes with bone turnover, which is thought to lead to necrosis. These drugs

\footnotetext{
${ }^{1}$ Specialist Registrar, ${ }^{2}$ Specialist Registrar, ${ }^{3}$ Consultant, ${ }^{4}$ Consultant, ${ }^{5}$ Consultant, Maxillofacial Surgery, New Cross Hospital, Wednesfield Road, Wolverhampton, WV10 00P

${ }^{*}$ Correspondence to: Mr Dilip Srinivasan

Email:godilip@yahoo.co.uk
}

can also induce tumour cell apoptosis and inhibit tumour invasion. In health the jaws have a greater blood supply and faster bone turnover than other bones which allows them to respond to various insults. Disruption of this innate ability and the presence of chronic dental disease makes the jaws uniquely susceptible to osteonecrosis. ${ }^{1,2}$

Osteonecrosis of the jaws was first reported in 2003 by Marx. ${ }^{3}$ Since then many case series have been published..$^{4,5}$ In spite of the fact that bisphosphonates have been in clinical use for a long period it is only now that this complication is becoming more apparent. A recent systematic review identified 368 reported cases in the literature. ${ }^{1}$ While the majority of cases followed dental treatment in one large series, 39\% of cases occurred spontaneously. ${ }^{2}$ The reported prevalence of ONJ in patients with cancer (who are on bisphosphonates) is between $6 \%$ and 10\%. ${ }^{1}$ The risk of developing ONJ increases with increasing dose and duration of bisphosphonate therapy. ${ }^{1,6}$

While all our patients had received zolendronic acid, ONJ has been reported following other intravenous and oral bisphosphonate therapies. ${ }^{4}$

\section{CASE 1 (Fig. 1)}

A 61-year-old male was referred with two months' lower jaw pain, difficulty opening his mouth and a foul taste. His past medical history included multiple myeloma for which he was receiving monthly zolendronic acid infusions.

Examination showed a discharging sinus in an edentulous mandible. Dental panoral tomograph (DPT) was unremarkable. Bone biopsy revealed necrosis. A diagnosis of ONJ was made and he was started on long-term tetracycline and mouthwashes. His pain was controlled, although a repeat DPT several months later showed extensive destruction.

\section{CASE 2}

A 53-year-old female was referred for a painful non-healing extraction socket. She had undergone simple extraction of a lower molar for pain. Her past medical history included breast cancer with skeletal metastasis for which she had received five infusions of zolendronic acid and oral clodronate.

Examination showed exposed bone in the mandible where the tooth had been extracted. DPT demonstrated areas of osteolysis with osteosclerotic sequestra. 


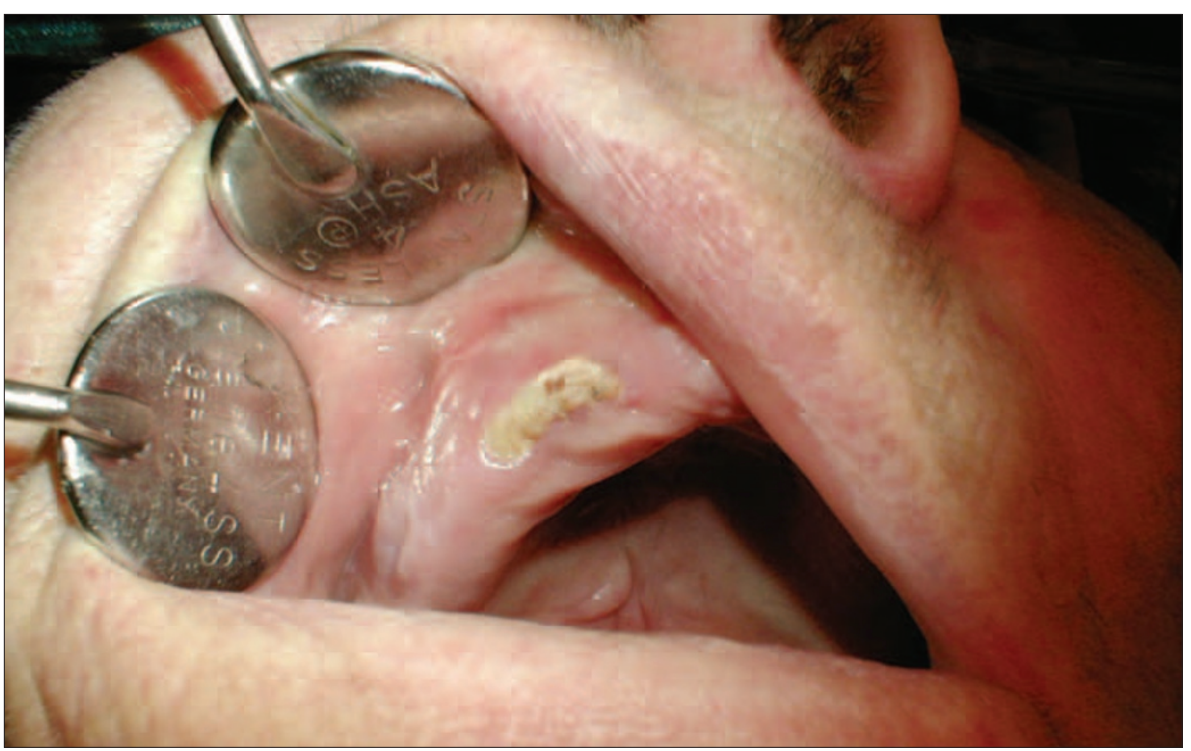

Fig. 1 Osteonecrosis of maxilla

She was treated with long-term antibiotics and mouthwash. Her bisphosphonates were stopped and she continues to have exposed bone but remains symptom free.

\section{CASE 3}

A 70-year-old female was referred for pain under her full upper denture. Her past medical history included metastatic breast cancer for which she received monthly zolendronic acid infusions.

Examination showed three areas of exposed maxilla under the upper denture. Biopsy confirmed ONJ. She is being treated with long term antibiotics (tetracycline) and mouthwash (chlorhexidene).

\section{DISCUSSION}

A high index of suspicion of ONJ is necessary in patients presenting with oral complaints on bisphosphonate treatment. Our cases demonstrate that the main feature of ONJ is pain, with or without the presence of teeth or a preceding surgical intervention. It should be noted that in about one third of reported cases pain was not a presenting feature. ${ }^{1}$ Early in the course of ONJ there are scant clinical findings. Hence there is a risk of not correctly diagnosing the cause of pain and attributing it to other well established causes. This could lead to erroneous treatment and worsening of the situation. Bone biopsy shows necrotic bone and chronic inflammation. Surgical interference may promote the bony spread of the condition but bone biopsy is justifiable to rule out primary and secondary tumours. Radiographic changes are absent early in the course of the disease. In established cases radiographic changes include a moth eaten appearance and radiolucency with or without radio opaque sequestra. This was seen in case one in our series.

Current guidelines for management of established osteonecrosis includes long-term antibiotics and chlorhexidene mouthwash. ${ }^{2}$ Surgical debridement should be restricted to refractory cases. Our cases have been managed using a conservative approach. Even though bony coverage by soft tissue has not occurred in all of our cases, our patients' symptoms are controlled. The half-life of bisphosphonates in bone can be as much as ten years but the anti-tumour effect of the drug demands continuation of therapy. Stopping the drug (if otherwise useful) is not therefore recommended. However, in patients with stable systemic disease some authors recommend withdrawal of bisphosphonates until the oral cavity symptoms improve. ${ }^{1}$

\section{CONCLUSION}

Bisphosphonate treatment is of great benefit to some cancer patients. ONJ following bisphosphonate therapy remains uncommon and symptomatic control can usually be achieved.

It is strongly advised for these patients to have a dental assessment and necessary treatment prior to starting bisphosphonate therapy. This approach of prevention is thought of as the best form of management. ${ }^{6}$

If dental health cannot be maintained following the administration of these drugs and an extraction becomes necessary then a referral to a specialist unit may be prudent.

1. Woo S-B, Hellstein J W, Kalmar J R. Systematic review: bisphosphonates and osteonecrosis of the jaws. Ann Intern Med 2006; 144: 753-761.

2. Marx R E, Sawatari Y, Fortin M et al. Bisphosphonate-induced exposed bone (osteonecrosis/osteoporosis) of the jaws: risk factors, recognition, prevention, and treatment. J Oral Maxillofac Surg 2005; 63: 1567-1575.

3. Marx R E. Pamidronate (Aredia) and Zolendronate (Zometa) induced avascular necrosis of the jaws: a growing epidemic. J Oral Maxillofac Surg 2003; 61: 1115.

4. Rugerio SI, Mekrota B, Engroff S L. Osteonecrosis of the jaws associated with the use of bisphosphonates. A review of 63 cases. J Oral Maxillofac Surg 2004; 62: 527.

5. Migliorati C E. Bisphosphonates and oral cavity avascular bone necrosis. J Clin Oncol 2003; 21: 4253 .

6. Migliorati C A, Casiglia J, Epstein J et al. Managing the care of patients with bisphosphonate-associated osteonecrosis: an American Academy of Oral Medicine position paper. J Am Dent Assoc 2005; 136: 1658-1668. 